\title{
Teindre au bleu de Prusse, une aventure française
}

\section{Prussian blue, such a French dye}

\author{
François Delamare ${ }^{1}$ \\ ${ }^{1}$ francois.delamare@gmail.com
}

RÉSUMÉ. Au milieu du XVIII ${ }^{e}$ siècle, le chimiste Macquer découvre que le bleu de Prusse, pigment récemment inventé, peut servir de colorant et teindre en bleu soie et laine. Mais il n'arrive pas à provoquer l'intérêt des teinturiers. Trente ans après, le projet est repris par Le Pileur d'Apligny, qui n'arrive pas à de meilleurs résultats. II faut attendre encore trente années et le Blocus continental pour que cette innovation soit jugée stratégique et digne de remplacer l'indigo qui n'arrive plus des colonies. La teinture au bleu de Prusse entre dans sa phase industrielle avec les Raymond père et fils. Elle remporte un grand succès par la beauté des bleus obtenus, et tient ce marché durant une cinquantaine d'année. Son déclin est lié à l'apparition des colorants de synthèse dérivés de l'aniline.

ABSTRACT. While Prussian blue did indeed begin its European career as a pigment, it was as a dye that it spread in France during the 19th Century. As early as the 18th century, various French chemists (Macquer, Le Pileur d'Apligny) had foreseen and proved that Prussian blue could be used as a dye. This discovery did not interest French dyers, who at that time used indigo from the French Antilles which was very cheap. It wasn't until the break in international trade due to the Continental System (1806) that the French government urgently began to seek a replacement blue. J.-M. Raymond perfected the Prussian blue dyeing of silk (Raymond blue) in 1811 and his son, P. Raymond, that of wool cloth in 1822. The shades obtained were so highly prized that these dyes would be retained after the Empire's fall, when France would again import indigo, English this time, from the Carolinas. The fashion in France for Prussian blue dyeing was then the cause for the development of an industry for the production of cyanides and yellow potash prussiate, the major part of which was used for dyeing. Manufacturing processes evolved but this flourishing situation was brutally brought to an end in the 1860 s by the appearance of the first blue aniline dyes.

MOTS-CLÉS. Teinture, bleu de Prusse, Macquer, bleu Raymond, indigo.

KEYWORDS. Dyeing, Prussian blue, Macquer, Raymond blue, indigo.

\section{Teindre en bleu}

Teindre en bleu nécessite la mise en œuvre d'un processus complexe, mais que l'on maîtrise depuis des temps immémoriaux, la teinture à la cuve. Il est fondé sur le fait que le colorant, l'indigotine, n'est pas soluble dans l'eau. Des fermentations, puis un milieu réducteur vivant provoquent l'apparition de molécules incolores capables de réagir avec les fibres textiles. L'oxydation à l'air de ces molécules provoque l'apparition de la couleur bleue.

Le processus est complexe, mais c'est une teinture remarquable. Elle est grand teint, et très économique. En effet, la plante tinctoriale est d'origine locale. Les plantes qui peuvent servir de sources d'indigo sont extrêmement nombreuses. Leur nombre dépasse la centaine. Celles poussant dans les pays chauds, comme l'indigotier du Mexique (indigofera suffructicosa Miller) sont beaucoup plus riches en produits actifs. En Europe occidentale, il s'agit de la guède, vouède ou pastel (isatis tinctoria $L$.). Pauvre en colorant, mais se cultivant bien, elle fournit des bleus qui accusent une petite teinte verdâtre contre laquelle il convient de lutter. 
Cette teinture est économique aussi parce qu'elle ne nécessite pas de mordant ${ }^{1}$, et qu'elle se pratique à faible température : le bain (la cuve) se mène à $50^{\circ} \mathrm{C}$.

En France, depuis le Moyen-âge, les teinturiers de bleu appartiennent à la corporation des teinturiers du bon ou grand teint. Une profession dynamique, fortement encadrée par des règlements contraignants qui tendent à promouvoir la qualité et unifier les productions, mais qui se méfie comme de la peste des innovations. Pour obtenir chaque teinte, il y a un chemin connu, éprouvé, dont il ne faut pas s'écarter. Depuis l'Instruction générale pour la teinture des laines rédigée par ordre de Colbert (1671), les nuances de bleu autorisées sont normalisées. Elles sont au nombre de treize. En allant de la plus proche du blanc à la plus saturée en bleu, elles sont baptisées [ANO 71]:

bleu blanc, bleu naissant, bleu pâle, bleu mourant,

bleu mignon, bleu céleste, bleu de reyne, bleu turquin,

bleu de roy, fleur de guesde, bleu pers, aldego, \&

bleu d'enfer.

A chacune d'entre elles devait correspondre un échantillon et un mode opératoire.

A bien y regarder, les teintures à l'indigo présentent aussi des inconvénients majeurs. Avec le procédé classique de la teinture à la cuve, les bleus obtenus ont des teintes très solides, mais un peu ternes et peu variées. De plus, les cuves, milieux vivants, sont difficiles à conduire et peuvent «tourner », ce qui augmente les coûts. Si pour éviter ces écueils, on choisit de dissoudre l'indigo dans l'acide sulfurique, les opérations sont plus faciles et les bleus obtenus, dits de Saxe, ont des teintes plus vives. Mais les couleurs sont moins solides, et les fibres textiles vieillissent mal. Coupable innovation, indigne d'un teinturier du bon teint.

En outre, au XVIII ${ }^{\mathrm{e}}$ siècle, l'indigo consommé en France n'est plus indigène, mais importé des Antilles françaises, en particulier de $\mathrm{S}^{\mathrm{t}}$ Domingue. Il provient d'indigofera suffructicosa $M$. Son prix fluctue en fonction de la conjoncture internationale, mais il s'agit d'indigotine presque pure $(95 \%)$. Après l'avoir traité d'aliment du Diable, de drogue fausse et pernicieuse et de couleur corrosive et rongeante, nocive et dolosive, force est aux teinturiers d'admettre qu'il s'agit d'un colorant de haute qualité. Après deux siècles de lutte, en 1750, la défaite du pastel est consommée. On n'emploie plus que l'indigo d'importation.

Il y aurait donc un véritable intérêt économique à mettre au point une teinture grand teint fournissant des teintes nouvelles de bleu, et utilisant un colorant que l'on puisse fabriquer sur place. Mais la rigidité du système ne favorise guère les innovations. Nous en aurons bientôt la démonstration.

\section{La naissance de la Chimie tinctoriale : Macquer}

En 1749 paraît en France un mémoire titré: Sur une nouvelle espèce de Teinture bleue, dans laquelle il n'entre ni pastel, ni indigo [MAC 49]. Loin d'être un teinturier, son auteur, Pierre Joseph Macquer est titulaire à Paris de la chaire de Chimie au Jardin du Roi, et membre de l'Académie Royale des

\footnotetext{
${ }^{1}$ Mordant : produit chimique (souvent un sel métallique), qui renforce la fixation du colorant sur la fibre. La très grande majorité des teintures utilisant des colorants naturels nécessite l'emploi de mordants. Le plus réputé était l'alun, sulfate double d'aluminium et de potassium. La nature du mordant influe sur la teinte obtenue ainsi.
} 
Sciences. Comme son collègue Jean Hellot et, avant eux, Charles François du Fay, il a été chargé par l'illustre Compagnie de jeter les bases d'une Chimie tinctoriale.

La chose est d'importance car l'économie du pays est fondée sur les industries textiles. Cotons, lins, soies et surtout laines, il y a peu de villes en France qui ne file, qui ne tisse et n'exporte ses productions textiles. Ces industries sont très dynamiques, et toujours soucieuses de rattraper une Angleterre qui nous précède souvent en ce domaine.

Fils ou pièces de tissus, cette production est destinée soit au blanchissage (linge de corps, uniformes), soit à la teinture (ameublement, costumes). Et dans ce dernier domaine, il est impossible de vendre fil ou tissu non teint ou mal teint. De plus, la mode étant changeante, il faut créer de nouvelles teintes, dans le respect des Règles de la profession et les Édits royaux. Blanchissage et Teinture, c'est dans ces deux domaines que l'Académie royale des Sciences, inspirée par Colbert, va lancer des recherches afin de détecter les procédés les plus efficaces employés, de les étudier, les améliorer et les recommander. Et puis pour en inventer de nouveaux. Ces recherches sont donc liées à un domaine d'activités hautement stratégiques pour le royaume.

Voilà donc Macquer en charge d'une Chimie tinctoriale. Et il se passionne pour cette branche de la chimie appliquée. Pour le moment, il est frappé par le fait que la palette du teinturier lui semble nettement plus pauvre que celle du peintre. Ainsi pour les bleus. Le peintre dispose du smalt, de l'azurite, des cendres bleues, de l'indigo, de lapis-lazuli et du bleu de Prusse. Le teinturier n'a à sa disposition que le pastel qui donne des bleus verdâtres et l'indigo des Antilles qui fournit une teinte bleue violacée. Comment enrichir cette palette ? Le plus simple ne serait-il pas de le faire avec des pigments? Laissons-lui la parole :

J'examinai d'abord si la Peinture ne pouvoit pas communiquer à notre art [la Teinture] quelquesunes de ces belles couleurs dont elle se pare avec tant d'avantage, \& je travaillai dans cette vûe avec d'autant moins de scrupule que je savois bien que si la chose étoit possible, je ferois à la Peinture cette espèce de larcin, sans lui causer le moindre dommage, les arts pouvant se faire réciproquement les plus beaux présens, sans que celui qui donne, risque de s'appauvrir en aucune manière.

D'où sa question : peut-on teindre un textile avec un pigment ? Bien sûr que non, répond le chimiste contemporain. L'art de la teinture consiste à disperser et fixer fortement les molécules colorantes sur les fibres textiles. Il y a donc réaction chimique. La dispersion d'un pigment, c'est-à-dire d'une poudre au sein des fibres ne permet pas de réaction chimique entre les grains et les fibres. Elle ne peut donner qu'une coloration instable qui disparaîtra au premier lavage.

Mais Macquer n'a pas les idées aussi nettes que nous sur les deux échelles très différentes auxquelles se produisent ces deux phénomènes. Il s'appuie sur une théorie qu'Hellot a récemment publiée (1740) et qui explique la mécanique invisible de la teinture [HEL 50]. En deux mots, la voici : dans la cuve d'eau chaude contenant le textile à teindre et le colorant, les micropores des fibres textiles se dilatent, s'ouvrent et laissent pénétrer les particules colorées. Puis le mordant agit comme un astringent, resserrant les micropores autour des particules colorées, qui se retrouvent enchâssées comme une pierre dans le chaton d'une bague. Nous avons donc affaire à une théorie purement mécanique dans laquelle les liaisons chimiques, qui sont en réalité, les acteurs principaux, ne jouent aucun rôle. De plus cette théorie suppose l'existence de micropores sur les fibres textiles, micropores qui n'existent pas.

C'est en raisonnant juste à partir de cette théorie erronée que, par le plus grand des hasards, Macquer va obtenir le résultat qu'il attend. En accord avec cette théorie, se dit Macquer, pour être un colorant de grand teint, il faut et il suffit que les particules colorées soient assez petites pour entrer dans les micropores, et que l'effet astringent soit suffisamment marqué. Mais quel pigment choisir ?: 
En passant en revîe les différentes couleurs, ma vûe se fixa principalement sur le bleu de Prusse, couleur éclatante dont la Chymie vient d'enrichir la Peinture depuis quelques années, \& que nous voyons tous les jours seconder si heureusement l'outre-mer dans nos plus beaux tableaux.

Le bleu de Prusse se réduit facilement en une poudre impalpable qui entrera facilement dans les micropores. Sa force colorante est exceptionnelle et puis, il y a une certaine similitude entre les fabrications du bleu de Prusse et les opérations de teinture avec mordant [DEL 07].

Qu'est-ce donc au juste que ce bleu de Prusse ? Le «bleu de Prusse » est découvert à Berlin par Dippel et Diesbach en 1706. L'appellation Berlinisch blau est attestée à la Société Royale de Berlin dès 1709. Il est Ceruleum Prussiacum et Prussian blue à la Royal Society en 1724, et Bleu de Prusse à l'Académie des sciences dès 1725 . La recette originale, où l'on voit un mélange de sels minéraux fristouiller dans du sang de bœuf est plutôt bizarre et ne nous éclaire guère. Le bleu de Prusse est un composé de fer et de cyanure. Plus exactement d'ions ferreux $\left(\mathrm{Fe}^{2+)}\right.$ et ferriques $\left(\mathrm{Fe}^{3+}\right)$ en quantité égale reliés entre eux par des ions cyanures $\left(\mathrm{CN}^{-}\right)$.

C'est la raison pour laquelle, pour faire du bleu de Prusse, on commencera par un bain de rouille (qui apporte le fer) et une opération complémentaire qui apportera les cyanures. Par exemple grâce à du sang de bœuf, qui fournit l'azote des protéines animales.

Ces trois espèces (ions ferreux, ferriques et cyanures) s'associent, s'organisent, et forment des cristaux. Ces cristaux présentent une particularité qui nous intéresse beaucoup : celle d'absorber très efficacement la partie jaune du spectre de la lumière solaire. Ils nous apparaissent donc très fortement bleu foncé. Telle est la raison de leur emploi pour une teinture.

\section{La teinture au bleu de Prusse}

Une fois ce choix fait, Macquer procède très simplement, en teinturier. Après avoir choisi des écheveaux de différentes sortes de fibres (laine, soie, coton...), il les plonge dans une solution d'alun et de vitriol (c'est le mordançage), puis dans le mélange de prussiate. Les résultats sont très positifs pour le coton et la soie. Pour la laine, la couleur est inégale et le tissu est devenu rude au toucher. Il soumet ses écheveaux aux différentes épreuves de « débouilli ${ }^{2}$ » et constate que la teinture tient bien : elle est de bon teint. Voici le jugement qu'il porte sur sa teinture :

Premièrement, elle est aussi supérieure en beauté \& en éclat au bleu de pastel et d'indigo, que l'écarlate l'est au rouge de garance. Cette couleur, comparée avec les plus beaux bleus faits suivant la méthode ordinaire, les efface tellement qu'on a de la peine à croire que ces derniers soient teints en bleu.

Secondement, le bleu ordinaire ne teint que la superficie des étoffes foulées, \& ne pénètre point dans l'intérieur, d'où il arrive que les draps bleus montrent une corde blanchâtre quand ils commencent à s'user : la nouvelle teinture pénétrant l'étoffe dans toutes ses parties, n'aura pas cet inconvénient, \& les draps qui en seront teints pourront s'user jusqu'à être troués, sans avoir ce coup d'œil désagréable.

Troisièmement, elle est de bon teint pour la laine \& pour la soie, \& soûtient très-bien, comme je l'ai dit, le débouilli d'alun. La teinture écarlate ne soûtient pas mieux que la nôtre le débouilli de savon.

\footnotetext{
${ }^{2}$ Débouilli. Épreuve visant à mettre en évidence les qualités de Grand teint d'une teinture. Elle consiste à faire séjourner le textile teint dans des solutions aqueuses bouillantes susceptibles d'attaquer la teinture (alun, savon, acide, base...).
} 
L'épreuve la plus sûre pour les teintures étant l'action de l'air \& du soleil, j'ai exposé des échantillons de la nouvelle teinture en plein air, à l'ardeur du soleil, pendant les mois entiers de Septembre \& d'Octobre de l'année dernière, pendant lesquels les jours ont presque toujours été sereins, \& ces échantillons n'ont point été déteints (...)

Quatrièmement, elle n'altère en aucune manière la bonté des étoffes, pourvî qu'on prenne les précautions que j'ai indiquées; pour m'en assurer, j'ai suspendu à des fils teints suivant cette méthode, des poids que j'ai toûjours augmentés jusqu'à ce que le fil se rompît, \& le fil de cette expérience ne s'est cassé que lorsqu'il a commencé à soûtenir un poids qui faisait rompre le même fil avant qu'il êut été teint. (...)

Comme tout savant peu au fait des conditions économiques régnant dans le domaine qu'il étudie scientifiquement, Macquer est optimiste sur le caractère applicable de sa découverte. Plus averti, Hellot lui conseille, avant toute publication savante, de montrer ses échantillons au Contrôleur Général des Finances, Machault d'Arnouville. Macquer se rend donc à Fontainebleau où se trouve la Cour pour remettre ses échantillons à ce dernier, qui les montre à Rouillé, conseiller au Bureau du Commerce. Celui-ci les renvoie à Hellot, qui établit un rapport déposé le 22 octobre 1748 à ce Conseil, rapport certifiant la date de la découverte du procédé par Macquer. A défaut de brevet, qui n'existe pas encore en France, Macquer obtient donc l'équivalent de notre « enveloppe Soleau » qui permet à un inventeur de déclarer son invention, et d'y attacher son nom ainsi qu'une date certaine.

Le cheminement administratif de ces échantillons montre bien l'intérêt que le pouvoir royal porte aux problèmes de teinture, un domaine jugé stratégique pour l'économie du Royaume.

\section{Un certain abbé Ménon}

Une autre raison peut être avancée pour expliquer la démarche de Macquer visant à une reconnaissance officielle. Le 23 mars 1749 (soit vingt-quatre jours avant qu'il soumette son Mémoire), avait été lu à l'Académie Royale des Sciences un Mémoire d'un abbé Ménon, correspondant de cette académie qui avait, un temps, étudié le bleu de Prusse. Dans ce nouveau mémoire, il exposait les résultats de deux études [MEN 50]. La seconde, fort brève, concernait la teinture des tissus au bleu de Prusse.

Ménon part d'une autre idée que Macquer. Pour lui, puisque l'on mordance les tissus au fer et qu'on les teint en noir au gallate de fer, noir qui peut être affaibli en bleu foncé lorsqu'on le délaye à l'eau, on doit pouvoir teindre au bleu de Prusse. Ses essais ratent sur le coton, et marchent assez bien avec la laine quoique les teintes obtenues ne soient pas uniformes. La teinture s'avère beaucoup plus facile avec la soie. Et Ménon de conclure :

D'ailleurs on peut faire le bleu de Prusse à peu de frais, \& en tirer une couleur autant au dessus des bleus ordinaires, que l'écarlate l'emporte sur la teinture à la garance. Ces avantages sont bien capables de piquer l'émulation de ceux qui travaillent pour l'utilité publique \& le progrès des Arts.

On ne peut pas ne pas remarquer la simultanéité et les similitudes qui existent entre les travaux des deux chimistes. Identité des sujets, et même de certaines phrases, comme celle où l'on compare la supériorité du bleu de Prusse sur l'indigo à celle de l'écarlate sur la garance. Ou bien la remarque sur le toucher de la laine teinte au bleu de Prusse qui est rude. Qu'en penser?

La lecture des deux Mémoires ne laisse aucun doute. Celui de Ménon est peu clair. Il ne traite de la teinture au bleu de Prusse que dans les deux dernières pages, et ne donne aucun détail expérimental. $A$ contrario, les onze pages du Mémoire de Macquer sont entièrement consacrés à la mise au point de cette teinture. Son Mémoire est d'une parfaite clarté et montre admirablement la méthode employée 
par ce chimiste dans sa recherche. Macquer ne s'est certainement pas inspiré de Ménon. Mais il y a certainement eu communication entre eux, et Ménon a utilisé les résultats de Macquer sans le citer. La postérité ne s'y est pas trompée, qui a oublié Ménon.

On peut imaginer que Macquer n'a guère goûté l'indélicatesse de Ménon et a voulu se garder de tels actes pour l'avenir. Il croit donc que son procédé tinctorial sera adopté, au moins par quelques teinturiers. Il n'en est rien. Ce procédé, très novateur, ne suscite aucun intérêt de la part des teinturiers de bleu. Un indigo de grande qualité arrive en énormes quantités de nos colonies de $\mathrm{S}^{\mathrm{t}}$ Domingue et de la Guadeloupe. Son prix fluctue bien un peu, mais il est généralement très bon marché. L'importation d'indigo par Nantes, Bordeaux et La Rochelle tient alors le troisième rang en valeur avec le coton, après le sucre et le café. En 1771, Bordeaux reçoit 1800 tonnes d'indigo. C'est le premier rang des importations avant le sucre et le café. La filière marche à merveille, et il n'est pas question qu'on l'abandonne, même sur la recommandation d'un membre éminent de l'Académie Royale des Sciences.

C'est l'échec classique d'une innovation qui ne répond à aucun besoin industriel. Macquer en prend son parti. En savant consciencieux, il publie un mémoire titré : Sur une nouvelle espèce de Teinture bleue, dans laquelle il n'entre ni pastel, ni indigo [MAC 49]. Et la teinture au bleu de Prusse tombe dans l'oubli.

Mais Macquer n'a pas dit son dernier mot sur ce sujet. Il s'intéresse à la réactivité du bleu de Prusse avec les bases. On sait que le bleu de Prusse ne craint pas les attaques acides, mais est détruit par les bases. Cette étude remarquable constitue un progrès marquant dans la compréhension des procédés de fabrication du bleu de Prusse [MAC 56]. Elle démontre l'existence d'un composé (qui sera plus tard nommé prussiate jaune de potasse) qui serait un intermédiaire idéal pour la fabrication car il permet une fabrication à la température ordinaire. Ce composé se révélera d'une importance capitale lorsqu'il s'agira de teindre industriellement des fibres textiles au bleu de Prusse.

\section{L'intervention de Le Pileur d'Apligny}

Elle refait surface trente ans après (1778), lorsque Charles Le Pileur d'Apligny, un spécialiste incontesté des procédés de teinture, auteur de divers ouvrages réputés sur la question, reprend le procédé Macquer de teinture au bleu de Prusse et le perfectionne. Pourquoi à cette date et pourquoi Le Pileur? Aucun document ne nous éclaire sur cette résurrection. Mais il est clair que Le Pileur qui, contrairement à Macquer, connaît bien le milieu des teinturiers, juge cette teinture fort intéressante.

Heureusement pour nous, Le Pileur a la bonne idée de soumettre son procédé à l'examen critique d'un jury de chimistes. Et ceux-ci publient un rapport fort instructif [CAD 78]. Ils expliquent que les teintures à l'indigo présentent industriellement divers inconvénients majeurs. Avec le procédé classique, les bleus à la cuve ont des teintes très solides, mais un peu ternes. Les cuves sont difficiles à conduire et peuvent «tourner», ce qui augmente les coûts. Si l'on dissout l'indigo dans l'acide sulfurique, les bleus de Saxe obtenus ont des teintes plus vives, mais moins solides, et qui vieillissent mal. De plus, l'indigo est importé d'au-delà des mers, et son prix fluctue en fonction de la conjoncture internationale. D'où l'intérêt de mettre au point une teinture grand teint fournissant de beaux bleus en utilisant un colorant que l'on puisse fabriquer sur place. C'est à l'ensemble de ces critères que satisfait la teinture au bleu de Prusse.

Comparant le procédé Le Pileur à celui proposé par Macquer, les rapporteurs notent diverses améliorations, dont la plus notable est l'utilisation d'une potasse beaucoup plus saturée en matière colorante car combinée directement à du bleu de Prusse, et non pas à du sang. Relevant pourtant quelques faiblesses, ils proposent un procédé simplifié et amélioré. Puis, ils discutent des coûts. 
Le prix de revient de cette teinture au bleu de Prusse semble être pour le moment équivalent à celui de la teinture au bleu de Saxe, mais le prix du bleu de Prusse tombe tous les jours au moyen des Manufactures multipliées qui s'en sont établies, principalement à Paris (...). De ce côté, l'avenir semble donc s'annoncer prometteur.

Soyons juste. La teinture au bleu de Prusse n'est pas sans défauts. D'abord, elle n'est pas vraiment grand teint. Mais elle est loin d'être la seule dans ce cas. L'expérience montre que des améliorations sont toujours possibles. Plus grave, elle ne résiste pas aux milieux alcalins, ce qui est une caractéristique du bleu de Prusse. Or les lessives employées sont alcalines. La nouvelle teinture ne peut donc s'appliquer qu'aux tissus que l'on n'envoie pas à la lessive, comme les draps de laine, les tissus de soie et les velours de coton. La teinture au bleu de Prusse ne remplacera donc pas la teinture à l'indigo dans tous les domaines, mais seulement dans ceux ne nécessitant pas de milieux alcalins.

Il est vrai que cet inconvénient est contrebalancé par le fait que la teinture au bleu de Prusse fournit des couleurs superbes, qui vieillissent bien. Et contrairement à l'indigo, elle permet de varier les nuances. L'avis des rapporteurs est donc très favorable. Considérant que Macquer (qui a alors soixante ans) est le véritable inventeur du procédé, ils lui présentent le travail de Le Pileur ainsi que les améliorations qu'ils proposent. C'est d'après l'avis de M. Macquer, \& avec lui que nous réclamons les encouragements de la Société [libre d'Émulation] en faveur de M. Le Pileur d'Apligny.

Mais rien ne force les teinturiers de bleu à innover. La filière «teinture à l'indigo des Antilles » fonctionne à la satisfaction générale. Et rien ne laisse prévoir quelque dysfonctionnement. La profession se complait dans une trompeuse sécurité. Résultat: la teinture au bleu de Prusse retombe dans l'oubli. Elle y restera une autre trentaine d'années.

\section{Gérer la soudaine pénurie d'indigo}

Le réveil est rude. Car les guerres menées par la France depuis la Révolution rendent difficile l'importation de l'indigo des Antilles. Et vient le blocus maritime imposé par les Anglais (1806) qui la stoppe totalement. Or la France importe pour trente millions de francs d'indigo par an, ce qui représente entre 1000 et 1500 tonnes d'indigo, suivant les cours. Ce colorant fournit quand même à très bon compte la teinture bleue la plus solide et la moins salissante, universellement utilisée. Où s'en procurer maintenant ? Où trouver les 150 tonnes d'indigo nécessaires pour teindre les uniformes des 600000 soldats de la Grande Armée ? Ou bien par quoi les remplacer? Les prises de corsaires (Le corsaire français Le héros du Nord se montre particulièrement efficace dans ce domaine en arraisonnant des navires hollandais chargés d'indigo en 1810-1811), voire la contrebande, ne sauraient y suffire. En août 1810, coté Français, la décision est prise de laisser entrer dans l'Empire les produits coloniaux, dès lors frappés d'une lourde taxe $(45 \%)$. Le résultat est, bien sûr, un renforcement de la contrebande effrénée qui existe depuis belle lurette entre l'Angleterre et les états germaniques proches de la mer. Fin 1810, une gigantesque opération de fouille des maisons et des entrepôts de Francfort est menée par les Autorités françaises. Elle mobilise des milliers de soldats. Deux cent trente-quatre négociants sont constatés en possession de marchandises coloniales - en particulier de l'indigo n'ayant pas payé la taxe. Meyer-Amschel (père des cinq frères Rothschild) est parmi eux. Pour son stock d'indigo, il doit payer 20000 francs de droits [BOU 60].

Pour répondre à la crise qui touchait l'ensemble des produits tropicaux encore récemment importés, certains départements furent brusquement promus au rang de producteurs de produits de remplacement. Ce fut le cas, par exemple, de la Haute-Garonne qui avait autrefois si bien profité de la 
culture du pastel et du commerce international de la cocagne ${ }^{3}$. Mais les temps avaient changé. La Haute-Garonne s'était convertie à la culture du maïs, plus rentable, et les agriculteurs, guère tentés de revenir en arrière faisaient traîner les choses. Crétet, ministre de l'Intérieur (nous dirions de l'Industrie) avait beau intervenir à la Chambre de commerce de Toulouse, et les études, s'accumuler, rien n'y faisait [FOH 49].

C'est probablement pour débloquer la situation que, sur la proposition du chimiste Jean-Antoine Chaptal, Napoléon crée en juin 1810 trois prix de montants extraordinairement élevés (le montant des prix est couramment compris dans la fourchette 600-3000 francs) [BUL 10]. Le premier, de cent mille francs, récompensera celui qui trouvera le moyen d'extraire d'une plante indigène et d'une culture facile une fécule propre à remplacer l'indigo, quant au prix, à l'emploi, à l'éclat et à la solidité de la couleur. (Art. I ${ }^{\mathrm{er}}$ ). Un second prix du même montant est destiné à celui qui fournira un procédé propre à fixer une couleur végétale indigène sur la laine, le coton, le lin et la soie, de manière à remplacer l'indigo, aux conditions de l'article $I^{e r}$. (Art. 2). Des prix intermédiaires sont créés, au cas où le procédé nécessiterait quand même l'emploi d'indigo. Leur montant est proportionnel à la quantité d'indigo épargnée. Un troisième prix, d'une valeur de vingt-cinq mille francs, ira à celui qui fera connaître un procédé sûr et facile pour teindre la laine et la soie avec le bleu de Prusse, de manière à obtenir une couleur unie, brillante, égale et inaltérable par le frottement et le lavage à l'eau.

On voit que Chaptal avait connaissance, au moins, des travaux de Le Pileur d'Apligny. On peut imaginer l'impulsion que ce décret impérial donna, à la fois dans le monde agricole, dans celui des chimistes, des inventeurs et des teinturiers pour la mise au point des divers procédés. A Paris, Claude Louis Berthollet et Chaptal travaillent, chacun de leur côté, à optimiser l'extraction des indigoïdes des feuilles de pastel. L'administration organise dans tout l'Empire la diffusion des graines de pastel en provenance d'Italie du Nord et la remise en culture de cette plante, assortie de primes versées aux agriculteurs. En 1811, 14000 hectares sont ainsi remis en culture. Une École de pastel s'ouvre à Albi. Des recherches y sont entreprises pour extraire une fécule d'indigo la plus concentrée qu'il est possible. Et des fabriques d'indigo-pastel sont créées à Toulouse, Turin, Florence et Borgo San Sepolcro. Dès 1811, on obtient des résultats très encourageants, et l'amélioration des procédés ne cessera de progresser. Hélas, le temps va jouer contre elles et à la Restauration, la fin du blocus et le retour des importations d'indigo les voue à la disparition.

Que se passe-t-il du côté de la teinture au bleu de Prusse ? Parallèlement à ces efforts entourant la culture du pastel et son exploitation, des chimistes reprennent le procédé Le Pileur amélioré et s'efforcent de le faire passer à l'échelle industrielle. Finalement, deux procédés de teinture de la soie, très proches l'un de l'autre, restent en lice, pour le prix. Le premier a pour auteur le chimiste Jean Louis Roard. Mais comme il est nommé membre de la commission d'attribution du Prix, il se retire du concours [PIN 87]. Nous ne connaissons pas les détails de son procédé. Le second est dû à un ancien préparateur de chimie à l'École Polytechnique, Jean Michel Raymond qui occupe la chaire de chimie industrielle nouvellement créée à Lyon par Napoléon pour soutenir les Teintures de Lyon.

\section{Le procédé Raymond pour teindre la soie}

Voilà donc remis au goût du jour le procédé de teinture de la soie par le bleu de Prusse autrefois découvert par Macquer, perfectionné par le Pileur d'Apligny puis par les rapporteurs de son travail.

\footnotetext{
${ }^{3}$ Cocagne : boule faite de feuilles de pastel fermentées et séchées, de la taille d'une boule de pétanque. Les cocagnes ne contenaient guère que $3 \%$ d'indigo, mais constituaient le produit marchand que les environs de Toulouse et, en particulier, le Lauragais exportaient dans toute l'Europe occidentale.
} 
Soixante-deux ans ont passé depuis la publication de Macquer. C'est peut-être ce qui explique que personne n'y fasse allusion. Mais Raymond doit la connaître, puisqu'il cite le second travail de Macquer sur le bleu de Prusse.

Le procédé Raymond consiste à mordancer la soie au fer, puis à la tremper ensuite dans une solution acide de prussiate de potasse. Une série de bains et de lavages intermédiaires évacuent impuretés et acidités résiduelles. Un dernier avivage à l'eau ammoniaquée fait foncer la couleur. Le résultat est très satisfaisant. En revanche, Raymond reconnaît que son procédé teint plus difficilement la laine, qui déteint par frottement.

L'innovation qui distingue ce procédé de ses devanciers est l'emploi du prussiate de potasse. Macquer avait employé le sang de bœuf et le Pileur, le bleu de Prusse lui-même. Cette fois, le procédé répond à une demande industrielle forte et urgente. Il est donc accueilli avec un grand intérêt. Pour son bleu Raymond qui ne teint pour le moment que la soie, Raymond reçoit en 1811 le tiers du prix proposé à l'article 3 du décret impérial de 1810, soit 8000 francs.

Reste à mettre en œuvre le procédé. C'est Jean Michel Haussmann, le grand fabricant d'indiennes de Logelbach, « le premier teinturier de France » qui, toujours à l'affût d'innovations, le met le premier en œuvre à l'échelle industrielle. Ce qui ne va pas sans quelques tracas pour lui comme pour ses émules. Témoin ce jugement porté par le vérificateur de l'administration du Mobilier impérial sur un lampas teint par Lacostat à Lyon par le procédé Raymond, lui reprochant de ne pas garantir l'unité de ton, de nuances, de couleur de tout un meuble. Icy la nuance varie, on trouve un Avis au centre d'une pièce, qui avertie le tapissier du changement de nuance ce qui est pitoyable [COU 87].

Il faut croire que les difficultés sont rapidement surmontées puisque l'administration impériale prend soin de diffuser le procédé dans tous les départements de l'Empire où existent des teintureries en soie. Des brochures sont imprimées à cet effet à Paris, Lyon, Tours, Turin, Gênes, Florence et Krefeld. Les préfets sont chargés de les distribuer. En témoigne cette lettre manuscrite conservée aux Archives Nationales ${ }^{4}$ :

Aix-la-Chapelle, le 18 septembre 1811

Monseigneur,

J'ai reçu avec la lettre dont votre Excellence m'a honoré le 7 de ce mois les 60 exemplaires du procédé découvert par $M^{r}$ Raymont pour teindre la soie avec le bleu de Prusse. Je me suis empressé de les répandre dans les teintureries de soie du Département de la Roër [Ruhr]. Roër.

Je Suis avec Respect, Monseigneur, De Votre Excellence, Le très humble \& très obéissant Serviteur Le Préfet de la

A Son Excellence le Ministre de l'Intérieur, Comte de l'empire

Simultanément, le procédé est publié en détail par Raymond dans diverses brochures [RAY 11]. L'essentiel est repris dans le bulletin de la Société d'Encouragement pour l'Industrie Nationale [BUL 14].

L'avenir du procédé repose sur la possibilité de se procurer du prussiate de potasse, produit dont la production va se développer au fur et à mesure du succès de la teinture au bleu Raymond. Car d'ersatz d'indigo (ce qui n'a plus de raison d'être dès 1814), le bleu Raymond acquiert bientôt une flatteuse

\footnotetext{
${ }^{4}$ Archives Nationales, F12-2252.
} 
réputation dans l'industrie textile. Des commandes prestigieuses sont passées, tel ce paravent en velours de soie, brodé et broché d'or, réalisé par la maison Grand Frères, de Lyon, d'après un modèle de Saint-Ange, qui orne actuellement la chambre du Roi au Louvre (figure 1).

Cet état de choses n'est pas ignoré du Dictionnaire technologique : Le bleu Raymond est une découverte d'autant plus précieuse pour la teinture, qu'auparavant on ne connaissait aucun moyen d'obtenir cette nuance sur soie; car on sait que les beaux bleus foncés bon teint se font à la cuve, et qu'ils n'ont jamais d'éclat, et que les bleus de ciel qui s'obtiennent avec la dissolution d'indigo, ne peuvent jamais atteindre le ton du bleu de Prusse (figure 2 échantillons) [ROB 23].

En fin de compte, l'adoption de la teinture au bleu de Prusse par les Teinturiers est le seul succès industriel qui résultera du Programme de recherche imaginé et mis en action par Chaptal en juin 1810. Fait remarquable, se succès perdurera jusque vers 1860 .

\section{Teindre la laine : tel père...}

Revenons un peu en arrière. Teindre la soie, c'est bien, mais il ne faut pas oublier que c'est le drap de laine qui constitue la majeure partie des textiles à teindre. Pour vêtir ses troupes, le gouvernement se voit dans l'obligation de trouver une solution. Or la demande en uniformes militaires n'a jamais été plus grande qu'à cette époque. Il faut vêtir les Marie-Louise, ces jeunes conscrits des classes 18141815 appelés par anticipation en 1813. Ils le seront de drap de laine teint au bleu Raymond (rebaptisé pour l'occasion bleu Marie-Louise) à l'aide d'un procédé qui n'est pas complètement maîtrisé. Cela n'aura sans doute pas changé grand-chose à leur destin.

Avec la chute de l'Empire, l'année 1815 voit la fin du blocus maritime. Les importations d'indigo des Antilles ne peuvent reprendre car les indigoteries françaises ont toutes fait faillite et ce sont les indigoteries anglaises des Carolines, aux Etats-Unis, qui prennent le relais.

Côté bleu de Prusse, il faut croire que l'on avait teint des métrages importants de drap de laine en bleu Marie-Louise, car à la Restauration, les surplus de ces draps sont vendus à bas prix. Ils sont rachetés dans les campagnes françaises, en particulier dans la région de Quimper. Telle serait l'origine de la singularité du costume masculin de cette région qui comporte un gilet de drap bleu (figure 3). La chose parut si originale que le pays quimpérois lui-même prit la dénomination de pays glazig (de glaz, bleu en breton). ${ }^{5}$

Pour la laine, le procédé doit être perfectionné. La teinture au bleu de Prusse des draps de laine est étudiée par le persévérant Roard, alors Directeur des Teintures à la manufacture des Gobelins à Paris. Mais c'est Raymond fils qui met au point le premier procédé industriel viable.

En 1822, il adresse des échantillons à la Société Royale d'Agriculture, Histoire naturelle et Arts utiles de Lyon aux fins d'essais de débouillis [TIS 23]. Par un heureux hasard, un pharmacien lyonnais, Souchon, a, lui aussi, envoyé des échantillons de draps de laine teints au bleu de Prusse pour essais. La comparaison n'est guère favorable à Raymond fils, mais celui-ci renvoie une nouvelle série d'échantillons qui montrent que la plupart des problèmes ont été résolus. Souchon abandonne le concours, et monte s'installer à Paris où il se fera un nom dans la fabrication du prussiate de potasse.

Raymond fils se lance alors dans une active campagne de communication sur son procédé qu'il juge maintenant industriellement viable. Il présente des échantillons de drap teint au bleu de Prusse à la

${ }^{5}$ J. Le Minor, communication personnelle. 
Société d'Encouragement pour l'Industrie nationale, puis présente plusieurs pièces de drap bleu teints à l'Exposition des Produits de l'Industrie Nationale de 1823 qui se tient à Paris, au palais du Louvre [BUL 25]. Il s'y voit décerner une médaille d'argent. Un mémoire complet décrivant minutieusement le procédé applicable aux draps comme aux laines en toison paraît en 1828 [RAY 28]. Raymond met au point son procédé en travaillant avec Seguin et $C^{\text {ie }}$, une société qui fabrique et teint des draps de laine à Annonay[COT 07]. Appliquée à la laine, la teinture au bleu de Prusse prend le nom de bleu de France.

À l'Exposition de 1834 qui se tient à Paris, on constate que, du fait du développement de la teinture au bleu Raymond, le prussiate de potasse supplante désormais le bleu de Prusse comme produit marchand.

En ce qui concerne la teinture au bleu de Prusse, reçoivent des médailles :

- Souchon, à Paris, rue Bleue n ${ }^{\circ} 19$, teinture au prussiate de fer de draps et mérinos.

- Merle et Malartic, à $S^{t}$ Denis, atelier de teinture au prussiate de fer. Ils présentent deux coupes de casimir, ${ }^{6}$ l'une bleu clair et l'autre bleu foncé, d'un reflet superbe, quoique teintes sans indigo.

Ces derniers industriels sont gens avisés. Ils arrivent à convaincre le baron Thenard, Président du Comité des Arts chimiques de la Société d'Encouragement pour l'Industrie Nationale ainsi que différents autres membres de ce Comité de porter un costume taillé dans un drap de laine teint au bleu de Prusse par leurs soins. C'est un coup de maître. Les témoignages convergent : cette teinture résiste au moins aussi bien que l'indigo à l'usure, et que les coutures et autres parties des vêtemens exposés au frottement ne blanchissent pas, bien que les draps éprouvés fussent teint à la pièce. Une visite des établissements Merle finit de convaincre les commissaires de la Société de la qualité des teintures et de l'étendue du commerce pratiqué, mais ces industriels désirent garder secret leur procédé [BUL 37].

\section{Le procédé Raymond fils pour teindre la laine}

En quoi consiste ce procédé ? Après avoir apporté le fer par un bain préliminaire de pertartro sulfate de fer dit bain de rouille, la nouveauté consiste à apporter les ions cyanures par deux bains successifs dits bains de bleu. L'un de prussiate de potasse, et l'autre d'acide hydrocyanique (HCN) dont on élève la température jusqu'à l'ébullition. Suivent un foulage du drap au savon et un avivage.

Si l'on restreint la gamme des bleus que l'on peut obtenir à cinq nuances également distantes les unes des autres en saturation, pour teindre un kilogramme de laine, il faut approximativement 15 grammes de prussiate pour obtenir l'équivalent d'un bleu naissant, 40 grammes pour un bleu céleste, 65 pour un bleu turquin, 85 pour un bleu pers et 100 pour obtenir la nuance la plus saturée, l'équivalent du bleu d'enfer [RAY 28]. Cette échelle des saturations reprend, en la simplifiant, l'échelle des treize nuances de bleu fixées par l'Instruction pour les teintures de Colbert pour la teinture à l'indigo (1671).

Dernière difficulté à vaincre : l'obtention reproductible d'une teinte donnée. En effet, on avait beaucoup de mal à obtenir les nuances voulues en teinture au bleu de Prusse. Raymond se contentait d'insister sur l'importance du bain de rouille. Le nouveau directeur des teintures aux Gobelins, Michel Eugène Chevreul, montre qu'il suffit de maîtriser avec précision la quantité de fer utilisée pour le bain de rouille, en utilisant des solutions convenablement dosées [CHE 26]. De plus, Raymond analyse en

\footnotetext{
${ }^{6}$ Casimir: drap léger en laine fine, tissé par l'armure batavia, presque exclusivement employé pour les vêtements d'hommes. P. Larousse, Grand Dictionnaire Universel du XIX ${ }^{e}$ siècle, Paris, 1867.
} 
détail les coûts et montre qu'en dépit de sa complexité, la teinture au bleu de Prusse est plus économique que la teinture à l'indigo d'importation.

Progressant de manière continue, la teinture au bleu Raymond va désormais, et pour une quarantaine d'années, consommer la part la plus importante du prussiate de potasse. Et cela d'autant plus que l'on va bientôt mettre au point un procédé d'impression des étoffes au bleu de Prusse. Le bleu de Prusse ne jouera alors plus le rôle de colorant, mais de pigment (figure 3).

La fin du bleu Raymond est due à l'émergence des colorants de synthèse. En effet, c'est peu avant 1860 que débute en France la teinture en bleu de la soie par un colorant de synthèse, l'azuline. Le premier établissement à la pratiquer est la maison Guinon, Marnas et Bonnet de Lyon. L'azuline sera bientôt remplacée par le bleu de Lyon découvert par Girard et de Laire en 1860, puis par des colorants de synthèse de plus en plus performants et faciles à utiliser. En 1865, chez Guinon, Marnas et Bonnet, la teinture au bleu Raymond n'est plus qu'un souvenir [TUR 65]. Les teintureries de soie vont très vite s'adapter. La désaffection pour le bleu Raymond est rapide et totale, ou presque. Car il reste quand même employé (en particulier à Lyon) pour la teinture en noir de la soie, fonction pour laquelle il est apprécié pour sa capacité à donner du poids et faciliter la prise des tannins [MOY 80].

\section{En conclusion}

En fin de compte, nous avons retracé l'histoire - peu connue - d'un nouveau procédé de teinture. Issu d'une série de recherches scientifiques, durant une soixantaine d'années, il fut considéré comme inutile. Un conflit international le met sur le devant de la scène, comme produisant un matériau stratégique. Et une fois industrialisé, il fonctionne à la satisfaction générale durant cinquante années. Puis il disparaît du fait des progrès de la chimie et de l'émergence d'un nouveau domaine scientifique, la Chimie Fine qui exacerbe la concurrence.

Cette aventure scientifico-industrielle présente deux singularités. La première est qu'elle trouve son inspiration dans un modèle erroné, le modèle mécanique de Hellot qui explique la mécanique invisible de la teinture. Par le plus grand des hasards, les expériences réalisées par Macquer semblent le vérifier. En fait, une simulation du procédé Raymond par les méthodes modernes de la mécanique quantique conduit à une autre explication plus réaliste, en mettant en évidence les réactions chimiques qui se produisent au contact des fibres [DEL 08].

La seconde singularité est qu'elle est exclusivement franco-française. Elle trouve sa source dans un programme lancé par l'Académie Royale des Sciences de Paris. Le premier chimiste (Français) intervenant, Macquer, adosse sa recherche à la théorie du Français du Fay. Chacun des intervenants de cette chaîne de chimistes qui améliore le procédé est Français. Ils publient leur procédé sans provoquer l'intérêt des pays voisins riches en industries textiles. Le procédé est finalement mis au point par les deux Raymond, père et fils, aidés par Chaptal, Berthollet et Chevreul. Tous Français. Roard qui pourrait être un concurrent, est Français. Bien que le procédé ait été diffusé en Allemagne et en Italie, c'est surtout Lyon qui teint la soie au bleu de Prusse. Et - comble de l'ironie - ce sont des chimistes lyonnais, Girard et de Laire, qui vont porter l'estocade au procédé avec leur bleu de Lyon. 


\section{Légendes des illustrations}

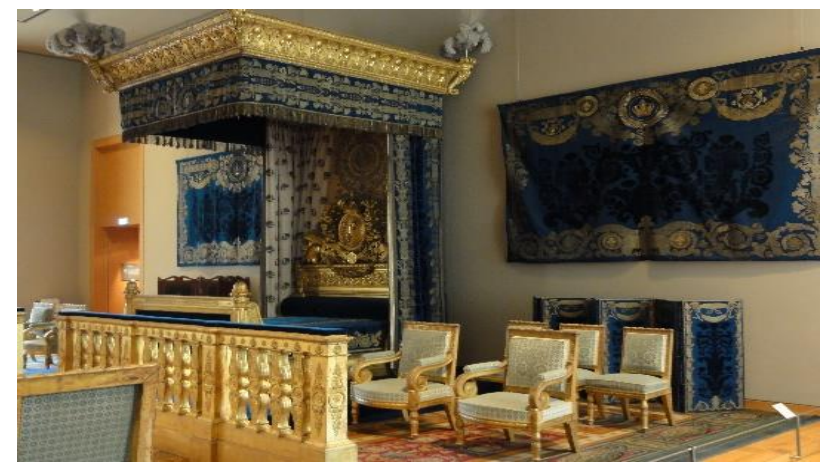

Figure 1. Chambre du roi Louis XVIII au palais du Louvre. Parure de soie teinte au bleu de Prusse par Grand, à Lyon, vers 1818. Cliché N. Delamare.

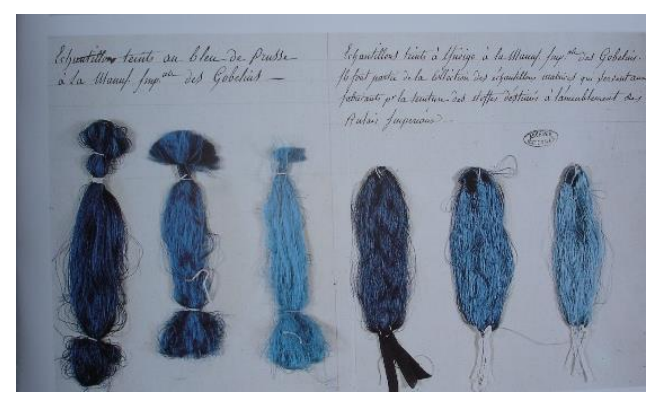

Figure 2. Échantillons de soie teints au bleu de Prusse et à l'indigo à la manufacture des Gobelins (1813). D’après Sublime indigo, Savants et Teinturiers, $n^{\circ} 182$, Fribourg, 1987.



Figure 3. Mathurin Méheut. Bretons du pays de Quimper portant la veste taillée dans un drap de laine teint au bleu de Prusse. Collection privée.

\section{Références}

[ANO 71] Anonyme, Instruction générale pour la teinture des laines etc., Paris, 1671, p. 30.

[BUL 25] «Coup-d'œil sur l'état actuel de l'industrie manufacturière en France », Bull. SEIN, $1^{\text {ère }}$ série, 24, 252, 1825, pp. 186-191. 
[BUL 10] « Programme d'un Prix proposé par ordre de S. M. L'EMPEREUR et ROI, pour remplacer l'indigo dans les teintures, tant par les produits du sol français que par ceux de l'industrie », Bull. SEIN, ${ }^{\text {ère }}$ série, 9, 72, 1810, pp. 155159.

[BUL 14] « Description d'un Procédé inventé par M. Raymond, professeur de chimie à Lyon, pour teindre la Soie avec le Bleu de Prusse, d'une manière égale, solide et brillante », Bull. SEIN, $1^{\text {ère }}$ série, 13, 116, 1814, pp. 29-41.

[BUL 37] « Rapport sur des draps teints au bleu de Prusse, par MM. Merle, Malartic, Poncet et compagnie, à Saint-Denis, par M. Bussy », Bull. SEIN, $1^{\text {ère }}$ série, 36, 398, 1837, pp. 329-330.

[BOU 60] Bouvier Jean, Les Rothschild, Paris, 1960.

[CAD 78] L. C. Cadet de Gassicourt, A. L. de Lavoisier, du Chanoy, «Rapport fait à la Société libre d'Émulation sur une teinture en bleu », Observations sur la Physique, sur l'Histoire naturelle et sur les Arts, 11, 1778, pp. 526-536.

[CHE 26] Chevreul Michel-Eugène, «Procédé pour dégrader les nuances de la teinture en bleu de Prusse sur soie », Bull. SEIN, 1ère série, 25, 267, 1826, pp. 286-287.

[COT 07] Cotte Michel, Le choix de la révolution industrielle. Les entreprises de Marc Seguin et ses frères (1815-1835), Presses universitaires, Rennes, 2007, sp. pp. 48-49.

[COU 87] Coural Chantal, in Sublime indigo, nº181, Fribourg, 1987, p. 162.

[DEL 07] Delamare François, Le bleu de Prusse, un destin inattendu, in Bleus en poudres, de l'Art à l'Industrie, 5000 ans d'innovations, Mines ParisTech, 2007, pp.181-234.

[DEL 08] Delamare François, B. Monasse, Étude du procédé Raymond de teinture par le bleu de Prusse, travail non publié (2008).

[FOH 49] Fohlen Claude, «A propos du blocus continental : le pastel toulousain », Annales du Midi, t. 61, 7-8, 1949, pp. 413-421.

[HEL 50] Hellot Jean, Théorie chymique de la teinture des étoffes, Mémoires de Mathématique et de Physique tirés des registres de l'Académie Royale des Sciences. Premier mémoire, Du bleu, année 1740, Paris 1742, pp. 126-148; second mémoire Du rouge et du jaune, année 1741, Paris, 1744, pp. 38-71. Mémoires réunis dans L'art de la teinture des laines et des étoffes de laine en grand et petit teint avec une instruction sur les débouillis, Paris, 1750.

[MAC 49] Macquer Pierre Joseph, Mémoire sur une nouvelle espèce de Teinture bleue, dans laquelle il n'entre ni pastel, ni indigo, Mémoires de Mathématique et de Physique tirés des registres de l'Académie Royale des Sciences de l’Année 1749, Paris, 1753, pp. 255-265.

[MAC 56] Macquer Pierre Joseph, Examen chymique du bleu de Prusse, Mémoires de Mathématique et de Physique tirés des registres de l'Académie Royale des Sciences de l'Année 1752, Paris, 1756, pp. 60-77.

[MEN 50] Abbé Ménon, Second mémoire sur le bleu de Prusse, Mémoires de Mathématique et de Physique présentés à l'Académie Royale des Sciences par divers Sçavants, \& lûs dans les Assemblées (Sçavants étrangers), 1749, t. I, Paris, 1750, pp. 573-592.

[MOY 80] Moyret M., «Couleurs et teintures en usage dans l'antiquité comparées à celles en usage de nos jours », Le teinturier pratique, 2,1880 , pp. 15-18.

[PIN 87] Pinault Michel, Savants et teinturiers, in Sublime indigo, Fribourg, 1987, pp. 135-141.

[RAY 11] Raymond Jean Michel, Description raisonnée d'un Procédé sûr et facile pour teindre la Soie en bleu de Prusse, Paris, 1811.

[RAY 28] Raymond P. (fils), "De la teinture des Laines au moyen du bleu de Prusse », Annales de Chimie et de Physique, 39, 1828, pp. 44-77. Un résumé se trouve dans «Extrait d'un Mémoire sur la teinture des laines au moyen du bleu de Prusse par M. Raymond fils », Bull. SEIN, $1^{\text {ère }}$ série, 27, 292, 1828, pp. 312-316.

[ROB 23] Robiquet Pierre, entrée Bleu Raymond, in Dictionnaire technologique, ou nouveau dictionnaire universel des arts et métiers, et de l'économie industrielle et commerciale, par une société de savans et d'artistes, tome 3, Paris, 1823, pp. 231-232.

[TIS 23] Tissier, «Expériences de teinture avec le Bleu Raymond, soumises à la Société par MM. Raymond fils et Souchon », in L. F. Grognier, Compte rendu des travaux de la Société Royale d'Agriculture, Histoire naturelle et Arts utiles de Lyon, Lyon, 1823, pp. 122-132. 
[TUR 65] Turgan Julien, « Teinturerie de soie. Guinon, Marnas et Bonnet à Lyon », in Les grandes usines de France, tome 4, Paris, 1865, pp. 257-272. 